\title{
Resveratrol promotes apoptosis and G2/M cell cycle arrest of fibroblast-like synoviocytes in rheumatoid arthritis through regulation of autophagy and the serine-threonine kinase-p53 axis
}

Shu Li ${ }^{1}$, Jinfeng Du ${ }^{1}$, Haina Gan², Jinwei Chen ${ }^{1}$, Yang Zhou ${ }^{1}$, Jing Tian' ${ }^{1}$, Guanghui Ling ${ }^{1}$, Fen Li ${ }^{1}$

\author{
${ }^{1}$ Department of Rheumatology and Immunology, The Second Xiangya Hospital, \\ Central South University, Changsha, Hunan, China \\ 2Department of Rheumatology and Immunology, The First People's Hospital \\ of Changde City, Changde, Hunan, China
}

Submitted: 17 November 2019

Accepted: 15 March 2020

Arch Med Sci 2020

DOI: https://doi.org/10.5114/aoms/119022

Copyright $\odot 2020$ Termedia \& Banach

\begin{abstract}
Introduction: Resveratrol, a polyphenol extracted from many plant species, has emerged as a promising pro-apoptotic agent in various cancer cells. However, the role of resveratrol in cell proliferation and apoptosis of fibroblast-like synoviocytes in rheumatoid arthritis (RA-FLS) is not fully understood.

The study was aimed at elucidating the role of resveratrol in cell proliferation and apoptosis of RA-FLS and the underlying molecular mechanism.

Material and methods: Cultured RA-FLSs were subjected to tumour necrosis factor $\alpha(T N F-\alpha)$. The cell proliferation was measured by Cell Counting Kit8 assay. Cell apoptosis and cell cycle of RA-FLSs were determined by flow cytometry. The levels of apoptosis or autophagy or cell cycle-related protein were detected by immunoblot analysis.

Results: In our study, we confirmed that resveratrol reversed TNF- $\alpha$ mediated cell proliferation in RA-FLS. Meanwhile, resveratrol blocked cells at the G2/M stage and reduced the ratio of $S$ phase cells through upregulation of $p 53$ and consequently led to apoptotic cell death. Quite interestingly, we found that resveratrol reversed TNF- $\alpha$-induced autophagy. Inhibition of autophagy by resveratrol or autophagy inhibitor or Beclin-1 siRNA suppressed TNF- $\alpha$ mediated cell survival and promoted cell apoptosis. However, the autophagy inducer rapamycin (RAPA) reversed the effect of resveratrol on autophagy and cell proliferation. Mechanistic studies revealed that resveratrol inhibited the activation of the phosphoinositide 3-kinases/serine-threonine kinase (PI3K/AKT) pathway. Inhibition of PI3K/AKT pathway by inhibitor LY294002 or resveratrol increased the expression of $\mathrm{p} 53$ and decreased the expression of cycle protein (cyclin B1), which further led to block cells in the G2/M arrest. Conclusions: Our preliminary study indicated that resveratrol may suppress RA-FLS cell survival and promote apoptosis at least partly through regulation of autophagy and the AKT-p53 axis.
\end{abstract}

Key words: resveratrol, rheumatoid arthritis, cell apoptosis, autophagy, p53.

\section{Introduction}

Rheumatoid arthritis (RA) is one of the most common inflammatory autoimmune diseases characterised by synovial hyperplasia and chronic

\author{
Corresponding author: \\ Prof. Fen Li MD, PhD \\ Department of Rheumatology \\ and Immunology \\ The Second Xiangya Hospital \\ Central South University \\ No.139 Ren Min Middle Road, \\ Changsha, Hunan 410011, \\ China \\ E-mail: lifen0731@csu.edu.cn
}


joint inflammation, which lead to adjacent cartilage destruction and progressive bone erosion [1]. Tumour-like hyperplasia of fibroblast-like synoviocytes (FLS) has been well demonstrated as a major role in the pathological process of RA [2]. Tumour necrosis factor $\alpha$ (TNF- $\alpha$ ) is regarded as one of the most central pro-inflammatory cytokines that significantly triggers the aberrant growth of fibroblast-like synoviocytes in rheumatoid arthritis (RA-FLS) and synovial inflammation [3]. Although treatment with disease-modifying antirheumatic drugs and biological agents have significantly improved the prognosis of patients, only a few patients achieve remission, and some patients are intolerant to such drugs $[4,5]$. Therefore, it is important to explore new therapeutic targets and drugs for RA.

Resveratrol, a trans-3,4',5-trihydroxystilbene, is a natural polyphenol compound found in various plants, vegetables, and fruits. Resveratrol has been reported to have a cytotoxic effect on tumour cells without affecting normal cells and has emerged as a promising antitumour agent [6]. Numerous studies have verified that resveratrol has a remarkable efficacy in blocking tumour progression and metastasis $[7,8]$. p53 is a tumour suppressor and plays a pivotal role in preventing tumour development by inducing cell cycle arrest at G0/G1 or $\mathrm{G} 2 / \mathrm{M}$ stage and/or promoting cell apoptosis in response to genotoxic stress $[9,10]$. Evidence shows that resveratrol blocked cell cycle at G0/G1 or G2/M phase and induced cancer cell apoptosis through upregulation and phosphorylation of p53 [11-13]. Moreover, in recent years, resveratrol has been reported to have an impact on the cell survival by regulating the expression of autophagy-related proteins and the autophagy signalling pathway $[14,15]$. Although great progress has been made in understanding the underlying molecular mechanisms of resveratrol in inhibiting cell proliferation and inducing cell apoptosis in various melanoma cells, the role of resveratrol in RA-FLS cell proliferation and apoptosis and its underlying molecular mechanisms have not been completely elucidated.

In the present study, we aimed to investigate the role of resveratrol in TNF- $\alpha$-induced autophagy and tumour-like proliferation of RA-FLS. We also explore whether resveratrol could inhibit TNF- $\alpha$-induced cell proliferation or promote cell apoptosis via activation of p53 and the underlying mechanism by which resveratrol regulates the p53 pathway.

\section{Material and methods}

\section{Reagents and antibodies}

Reagents and antibodies were obtained from the following sources: recombinant TNF-a, res- veratrol (Res),3-Methyladenine (3-MA), phosphoinositide 3-kinases (PI3K) inhibitor LY294002, chloroquine (CQ), rapamycin (RAPA), and dimethyl sulphoxide were purchased from Sigma-Aldrich (St. Louis, MO). Anti-Beclin-1,anti-LC3, anti-PI3K, anti-phospho-Akt,anti-Akt,anti-caspase3, anti-p53, anti-cycle protein and anti- $\beta$-actin were from Cell Signaling Technology (Beverly, MA); Horseradish peroxidase (HRP) conjugated anti-rabbit IgG, and HRP-conjugated anti-mouse IgG were from Cell Signal Technology (Beverly, MA). Beclin-1-siRNA(5'-CAGUUACAGAUGGAGCUAATT-3') and control scrambled siRNA(5'-CUUACGCUGAGUACUUCGATT-3') were obtained from GENE (Shanghai, China). Neon ${ }^{\mathrm{TM}}$ Transfection System, foetal bovine serum (FBS), Dulbecco's modified Eagle's medium (DMEM), and other materials for cell culture were from Invitrogen (Karlsruhe, Germany).

\section{Cell culture}

Synovial tissues were obtained from 6 active RA patients undergoing knee joint synovectomy or replacement (5 women, 1 man, aged 55-72 years). All patients were diagnosed according to the revised criteria of the American College of Rheumatology [16]. The study was approved by the Ethics Committee of XiangYa Second Hospital, Central South University (Hunan, China), and all patients provided written informed consent (ethical batch number: 2017-LS-S036). Fibroblast-like synoviocytes (FLS) were isolated as previously reported [17]. Cells were cultured in DMEM with $15 \%$ FBS under an atmosphere of $5 \% \mathrm{CO}_{2}$ and $95 \%$ air at $37^{\circ} \mathrm{C}$. Cells from passages 2 to 6 were used in the study.

\section{Cell treatment}

Fibroblast-like synoviocytes in RA were grown to approximately $80 \%$ confluence and subjected to serum-deprivation for 24 hours before experimental manipulation. Cells were treated with resveratrol or autophagy inhibitors or autophagy inducer or PI3K inhibitor LY294002 before TNF- $\alpha$ exposure. After 24-hour incubation, cells were collected for further analysis.

\section{SiRNA transfection}

Beclin-siRNA and control scrambled siRNA were transfected into RA-FLS at $80 \%$ confluent using a Neon ${ }^{\mathrm{TM}}$ Transfection System. Briefly, confluent cells were trypsinised and resuspended in resuspension buffer at a density of $2 \times 10^{5}$ cells per $10 \mu \mathrm{l}$ of solution; $50 \mathrm{nM}$ Beclin-siRNA and control scrambled siRNA were added separately. Cells were transfected by electroporation according to the manufacturer's instructions. 


\section{Analysis of cell proliferation}

A Cell Counting Kit-8 (CCK-8) assay kit was used to determine the cell viability according to the manufacturer's instructions. $10 \mu \mathrm{l}$ CCK-8 reagent was added to each well and the cells were incubated for $1 \mathrm{~h}$ at $37^{\circ} \mathrm{C}$ and $5 \% \mathrm{CO}_{2}$. The absorbance of optical density was measured at $450 \mathrm{~nm}$.

\section{Flow cytometry}

The rate of cell apoptosis and cell cycle of FLS were assessed by flow cytometry (fluorescence-activated cell sorting). In brief, FLS cells suspension were washed, trypsinised, and collected by centrifugation, and then stained with Annexin V-FITC and propidium iodide $(\mathrm{PI})$ by using the cell apoptosis detection kit. The percentage of apoptotic cells and the proportions of cells in G0-G1, S, and $\mathrm{G} 2 / \mathrm{M}$ phase were determined by flow cytometric analysis.

\section{Western blotting}

Total protein was extracted from RA-FLS using lysis buffer containing protease inhibitors. And all samples were separated by $10 \%$ SDS-PAGE and transferred onto PVDF membranes. The blots were incubated with specific antibodies against Beclin-1, LC3, PI3K, serine-threonine kinase (AKT), p-AKT, cycle protein (cyclin B1), p53, and cleavedcaspase 3 or $\beta$-actin, respectively. Expression of specific proteins was detected using an enhanced chemiluminescence system, and densitometric analysis was performed as previous described.

\section{Statistical analysis}

All experiments were repeated at least three times. The distribution of the statistical data was identified by Kolmogorov-Smirnov (K-S) test and the graphical assessment of normality. All data conformed to normal distribution and were expressed as mean \pm standard deviation. Statistical analysis was performed with Student $s$ t-test and one-way analysis of variance, and $p<0.05$ was considered statistically significant.

\section{Results}

Resveratrol reversed tumour necrosis factor $\alpha$-mediated cell proliferation and promoted cell apoptosis in fibroblast-like synoviocytes in rheumatoid arthritis cells

To address the role of resveratrol in RA synovial fibroblast proliferation and apoptosis, RA-FLSs were treated with $50 \mu \mathrm{M}$ resveratrol 1 hour before exposure to TNF- $\alpha$ (10 $\mathrm{ng} / \mathrm{ml})$. Cell viability and the rate of cell apoptosis or cell cycle of RA-FLSs were determined by CCK-8 and flow cytometric analysis, respectively. As shown in Figure 1, cell viability was significantly increased after TNF- $\alpha$ incubation for 48 hours (115.33\%) compared with the control group (100\%). Notably, treatment with resveratrol effectively reversed TNF- $\alpha$-induced cell proliferation (100.69\%). In addition, the rate of cell apoptosis was similar between the control group (8.33\%) and the TNF- $\alpha$ group (10.78\%). However, resveratrol treatment could augment cell apoptosis (29.08\%) compared with the TNF- $\alpha$ group. Our data indicate that resveratrol could suppress cell survival and trigger apoptosis in RA-FLS.

\section{Resveratrol promoted cell apoptosis by regulation of autophagy flux}

There is considerable evidence demonstrating that autophagy plays a dual role in either promoting cell survival or triggering cell death [18]. To elucidate the effect of resveratrol on autophagy flux, we treated cells with $50 \mu \mathrm{M}$ resveratrol. To ascertain whether resveratrol inhibited cell survival and promoted cell apoptosis by regulation of autophagy flux, cells were treated with autophagy inhibitor 3-MA (10mM) or CQ $(50 \mu \mathrm{M})$ or Beclin-1 siRNA $(50 \mathrm{nM})$ or autophagy inducer RAPA (10nM) before exposure to TNF- $\alpha(10 \mathrm{ng} / \mathrm{ml})$. The ratio of cell proliferation and apoptosis were determined by CCK-8 assay and flow cytometry kit separately. In the present study, our data clearly showed that resveratrol downregulated the expression of autophagy-related protein Beclin- 1 and attenuated the TNF- $\alpha$-induced LC3-I to LC3-II conversion, while increasing the expression of cleaved-caspase 3 (Figure $2 \mathrm{~A}$ and $B)$. Inhibition of autophagy by resveratrol or autophagy inhibitors (3-MA and CQ) or Beclin-1 siRNA significantly increased the percentage of cell apoptosis and suppressed cell survival compared to the TNF- $\alpha$ group (Figure $2 \mathrm{C}-\mathrm{G}$ ). However, autophagy inducer RAPA partly reversed the effect of resveratrol on cell autophagy and cell survival (Figure 2 $\mathrm{H}-\mathrm{I})$. Taken together, these findings suggest that TNF- $\alpha$-induced autophagy plays an important role in TNF- $\alpha$-induced cell apoptosis resistance, and resveratrol suppressed cell proliferation and promoted cell apoptosis at least partly through regulation of Beclin-1-related autophagy pathway.

\section{Resveratrol inhibited tumour necrosis factor $\alpha$ induced cell proliferation by regulating $\mathrm{p} 53$ and cell cycle in fibroblast- like synoviocytes in rheumatoid arthritis}

Ample evidence demonstrated that tumour suppressor protein $\mathrm{p} 53$ was involved in the RA pathological process $[3,19,20]$. However, whether p53 is involved in resveratrol-induced cell apoptosis of synovial fibroblasts is still unknown. In this study, the level of p53 and cyclin B1 and the ratio of cells 
A

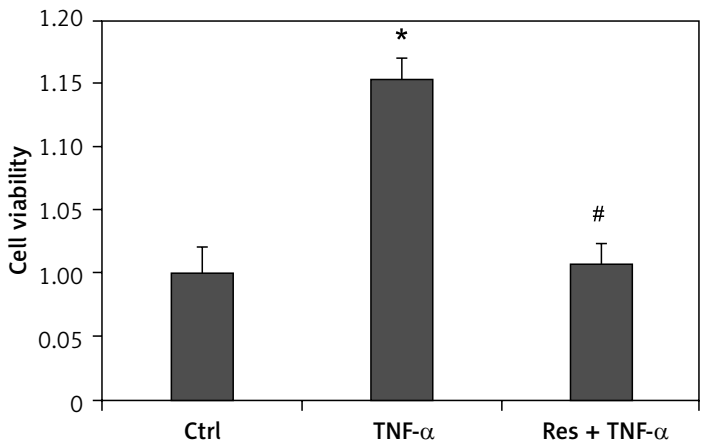

B

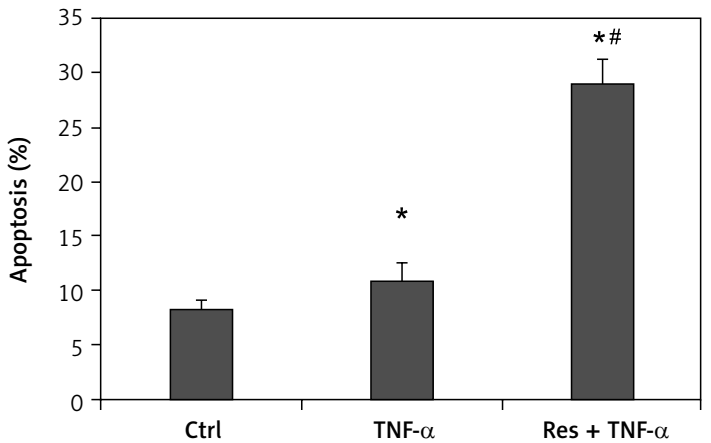

C
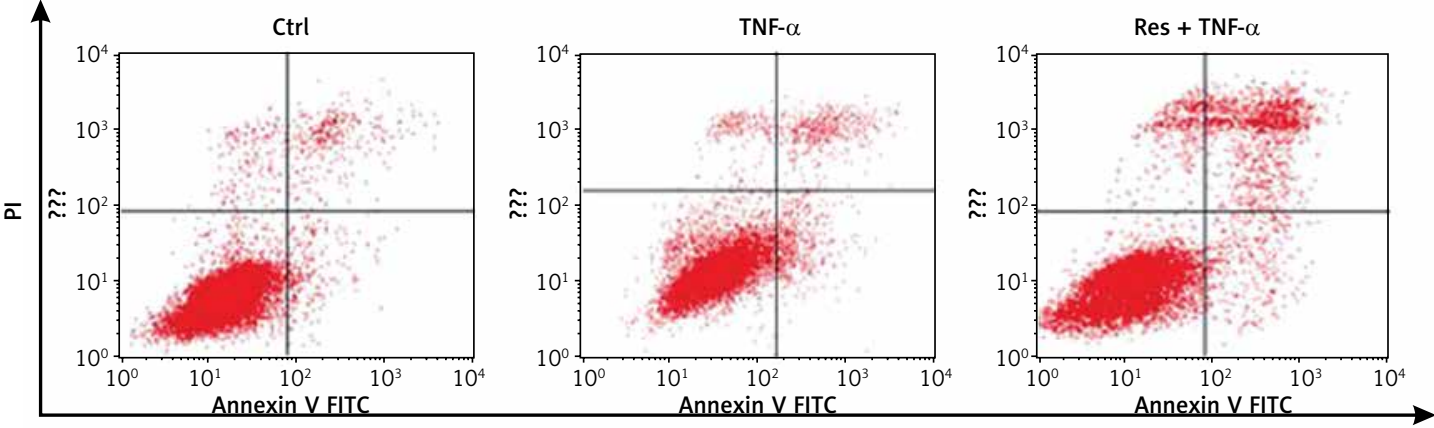

Annexin V

Figure 1. Effects of resveratrol on cell apoptosis and cell proliferation in fibroblast-like synoviocytes in rheumatoid arthritis (RA-FLS) cells induced by tumor necrosis factor $\alpha$ (TNF- $\alpha$ ). A - Cell proliferation was detected by Cell Counting Kit-8 method. Quantified histograms of cell viability of RA-FLS cells in different groups are shown in Figure 1A. B - Apoptosis cells were evaluated by flow cytometry using annexin V/propidium iodide staining. Quantified histograms of apoptosis RA-FLS cells in different groups are shown in Figure 1B. C-Representative images showed resveratrol treatment could augment celi apoptosis compared with the cells treated with TNF- $\alpha$ alone. Data are expressed as mean \pm SEM of separate experiments performed in triplicate

${ }^{*} p<0.05$ compared with control group. ${ }^{*} p<0.05$ compared with TNF- $\alpha$ group.

in different cell cycle stage after resveratrol treatment were evaluated by immunoblotting and flow cytometry methods, respectively. Our data showed that resveratrol upregulated the expression of p53 level and downregulated the cell cycle protein cyclin B1 level compared with TNF- $\alpha$-alone group (Figure $3 \mathrm{~A}$ and $\mathrm{B}$ ). Meanwhile, resveratrol could block cell cycle arrest at the G2/M phase, and the ratio of cells in $\mathrm{S}$ phase was obviously decreased (Figure $3 \mathrm{C}$ and $\mathrm{D}$ ). These findings revealed that resveratrol rescued RA-FLS cells from TNF- $\alpha$-induced proliferation by upregulation of p53 and downregulation of cell cycle protein cyclin B1, which further led to cells stagnated at the G2/M phase.

Resveratrol-induced cell cycle stagnated at $\mathrm{G} 2 / \mathrm{M}$ phase by regulation of the serinethreonine kinase-p53 axis in fibroblast-like synoviocytes in rheumatoid arthritis

To further elucidate the underlying molecular mechanism by which resveratrol inhibits TNF- $\alpha$ induced cell survival in RA synovial fibroblasts, the effect of resveratrol on the expression of AKT and phosphorylation of p-AKT was determined by western blot. Compared with the control group, TNF- $\alpha$ increased the expression of $p-A K T$ and AKT in RA-FLS. However, treatment with resveratrol abolished the effect of TNF- $\alpha$. Next, we investigated the effect of PI3K inhibitor LY294002 on the cell viability and the expression of cell cycle related proteins (p53 and cyclin B1). As shown in Figure 3, compared with TNF- $\alpha$ alone group, PI3K inhibitor LY294002 remarkably reduced the cell viability. Interestingly, we found that, compared with TNF- $\alpha$ group, resveratrol and PI3K inhibitor LY294002 treatment upregulated the expression of $\mathrm{p} 53$ and downregulated the expression of cyclin B1, further blocked cell cycle arrest at the G2/M phase, and decreased the ratio of $\mathrm{S}$ phase cells (Figure 3). Taken together, all these results suggest that resveratrol may induce cell arrest at the G2/M phase partly through regulation of the AKT/p53 axis.

\section{Discussion}

In this study, we demonstrated that resveratrol can effectively inhibit cell proliferation and pro- 
A
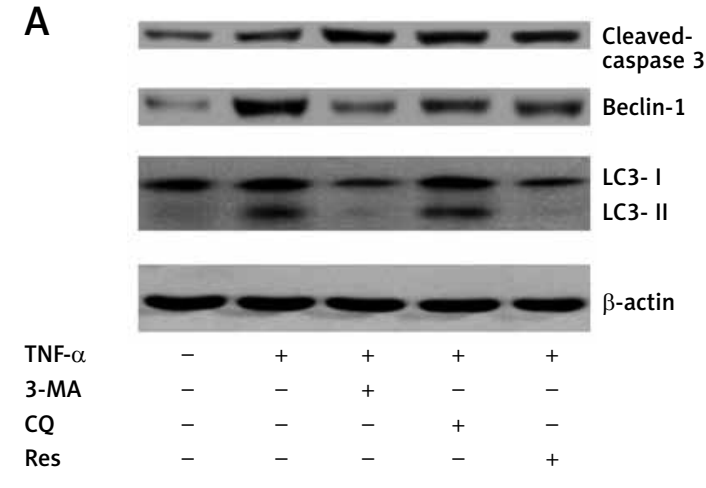

C

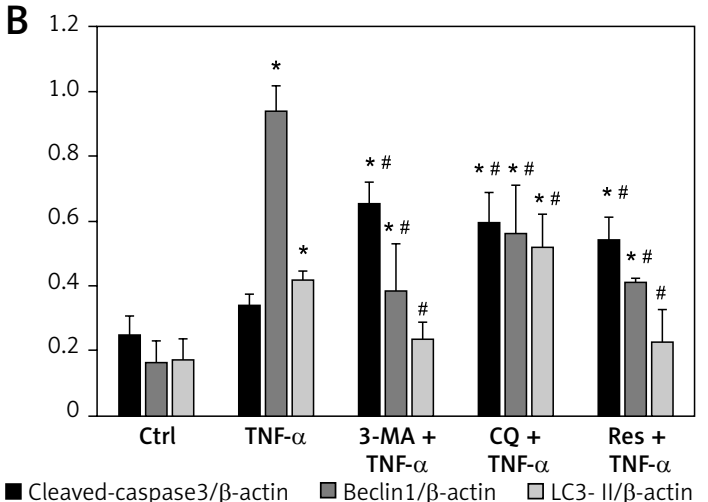

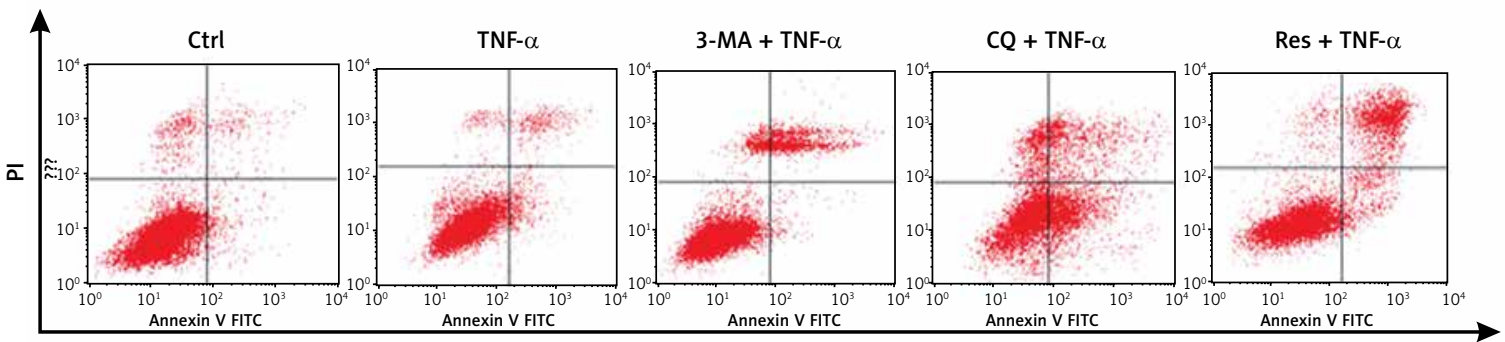

Annexin V
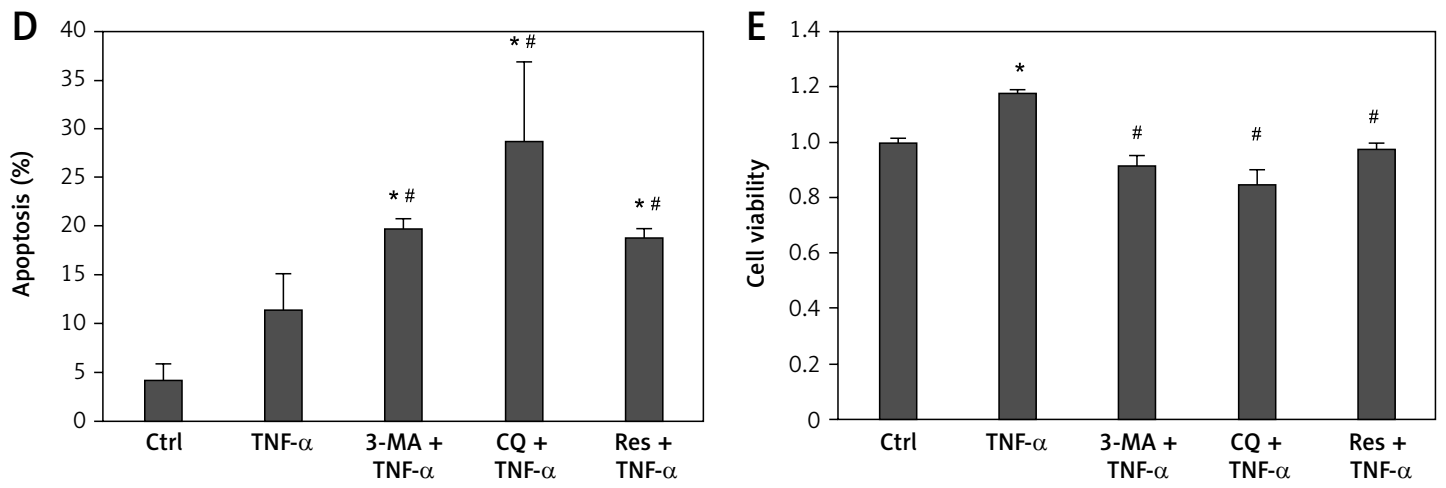

$\mathbf{F}$
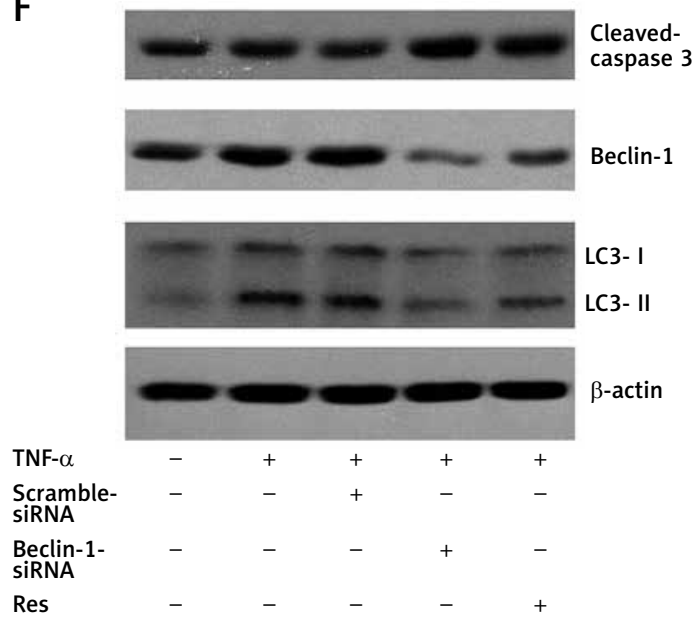

G

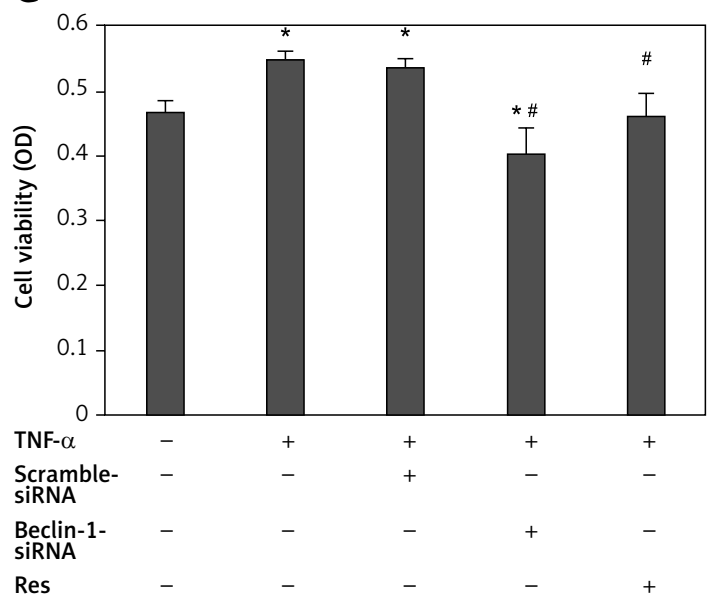

Figure 2. Resveratrol inhibited tumour necrosis factor $\alpha$ (TNF- $\alpha$ ) induced cell proliferation and promoted cell apoptosis by regulation of autophagy flux in fibroblast-like synoviocytes in rheumatoid arthritis (RA-FLS) cells. A - The effect of resveratrol on the expression of Beclin-1, LC3-I, LC3-II, and cleaved-caspase 3 were assessed by western blot method. B - Protein expression levels of the indicated proteins were quantitatively analysed by densitometry and normalised with $\beta$-actin. C - Apoptosis cells were evaluated by flow cytometry using annexin V/ propidiumiodide (PI) staining. D - Quantified histograms of apoptosis RA-FLS cells in different groups. E - Cell proliferation was detected by Cell Counting Kit-8 method. $\mathbf{F}$ - The effect of knockdown of Beclin-1 expression on autophagy and apoptosis. G - The effect of knockdown of Beclin-1 expression on cell proliferation

${ }^{*} p<0.05$ compared with control group. ${ }^{*} p<0.05$ compared with TNF- $\alpha$ group. ${ }^{*} p<0.05$ compared with Res + TNF- $\alpha$ group. 
$\mathrm{H}$

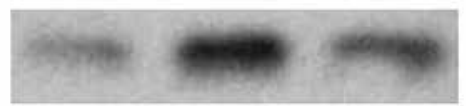

Cleavedcaspase 3
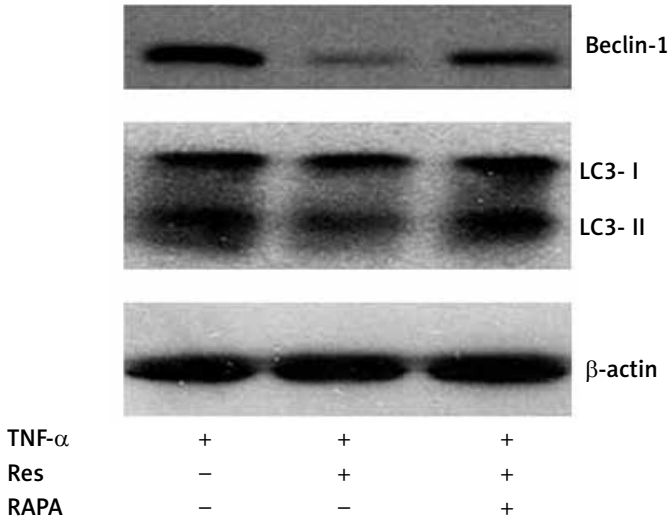

1

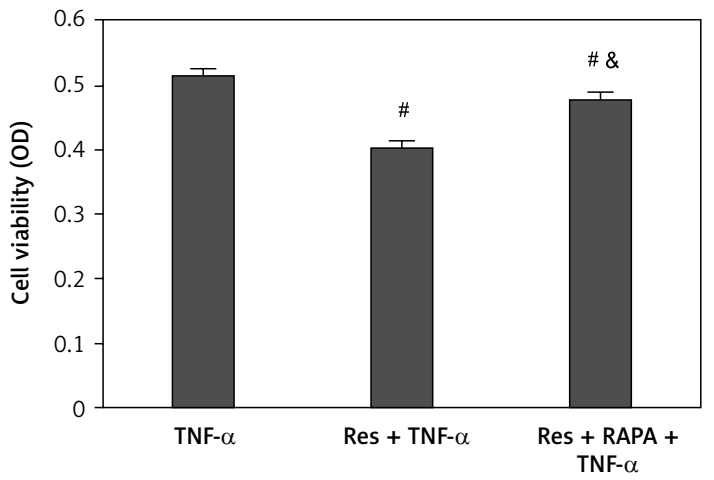

Figure 2. Cont. $\mathbf{H}$ - The effect of resveratrol on the expression of Beclin-1, LC3-II, and cleaved-caspase 3 was reversed by autophagy inducer rapamycin (RAPA). I - The effect of resveratrol on cell proliferation was reversed by autophagy inducer RAPA. Data are expressed as mean \pm SEM of 3 independent experiments

${ }^{\star} p<0.05$ compared with control group. ${ }^{*} p<0.05$ compared with TNF- $\alpha$ group. ${ }^{\circledR} p<0.05$ compared with Res + TNF- $\alpha$ group.

mote cell apoptosis by regulation of autophagy flux in RA-FLS. Simultaneously, resveratrol reversed TNF- $\alpha$-induced synovial fibroblasts proliferation by upregulation of p53. Moreover, resveratrol and PI3K inhibitor (LY294002) treatment concurrently decreased the expression of $\mathrm{p}-\mathrm{AKT}$ and AKT and downregulated the expression of cyclin B1, and further blocked cell cycle arrest at the G2/M phase. Our findings indicate that resveratrol abolishes the promotive effect of TNF- $\alpha$ on cell proliferation in RA-FLS by regulation of autophagy and cell cycle. Furthermore, as summarised in Figure 4 resveratrol suppresses cell survival and triggers cell apoptosis, at least in part, through downregulation of autophagy and the mechanism involved in PI3K/AKT pathway-mediated upregulation of p53 expression and downregulation of cyclin B1.

Numerous previous studies have demonstrated that resveratrol has antioxidant, anticancerogenic, and immunomodulatory properties. It is well documented that resveratrol has remarkable efficacy in inhibiting cell proliferation and promotes cell apoptosis in various tumour cells [21-23]. However, the role of resveratrol in regulating TNF- $\alpha$-induced tumour-like growth of synovial fibroblasts and its underlying mechanisms have not been fully illustrated. Autophagy is an evolutionarily conserved degradative process involved in clearance or turnover of misfolded proteins and damaged organelles. Autophagy is thought to be involved in a variety of pathological and physiological events. Ample evidence has clarified that autophagy plays a central role in cell survival, cell death, and intracellular homeostasis [24-26]. Recent studies suggest autophagy is a double-edged sword in tumourigenesis and metastasis. Autophagy can serve as a pro-survival or pro-death stress response in different conditions [27-29]. Recent evidence has shown that autophagy is involved in various immune processes and plays an important role in regulating the release of inflammatory cytokines and the pathological process of bone destruction in RA [30, 31]. Our previous study showed that the autophagy inhibitors 3-MA and CQ abolished the effect of TNF- $\alpha$-mediated apoptosis resistance in synovial fibroblasts [16]. However, it has never been investigated whether autophagy is involved in the mechanism of resveratrol-mediated synovial fibroblast apoptosis. In the present study, our findings showed that resveratrol treatment prevented TNF- $\alpha$-induced autophagy by downregulation of the expression of Beclin-1 and LC3-II in RA-FLS. Autophagy pharmacological inhibitors 3-MA and CQ or knockdown of the expression of Beclin-1 could reverse TNF- $\alpha$-induced cell proliferation and increase the expression of cleaved caspase 3 level and the percentage of cell apoptosis. Autophagy pharmacological inducer RAPA could partly reverse the effect of resveratrol on cell proliferation and apoptosis. Taken together, all these data suggest that resveratrol could inhibit TNF- $\alpha$-induced cell proliferation and promote cell apoptosis at least partly through downregulation of Beclin-1, further inhibiting TNF- $\alpha$-induced autophagy in RA-FLS.

p53, a tumour-suppressor protein, plays a central role in the induction of cell cycle arrest and cell apoptosis in response to diverse cellular stimuli. Accumulating evidence has demonstrated that p53 plays a crucial role in RA-FLS cell apoptosis [3, 20, 32]. p53 signalling pathway has become a promising target in the treatment of RA. In recent years, resveratrol has been demonstrated to trigger cell apoptosis in certain cancer types by 
A

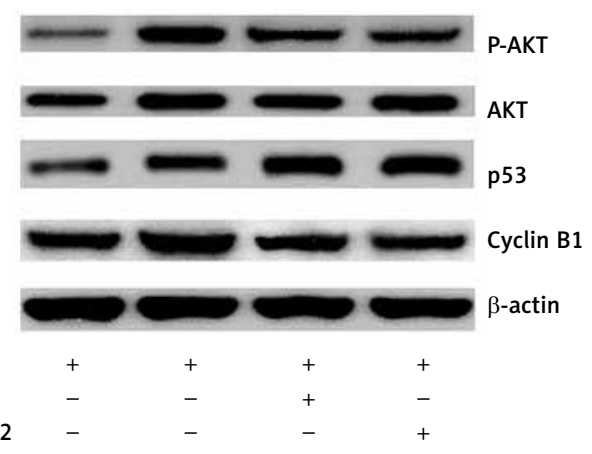

C

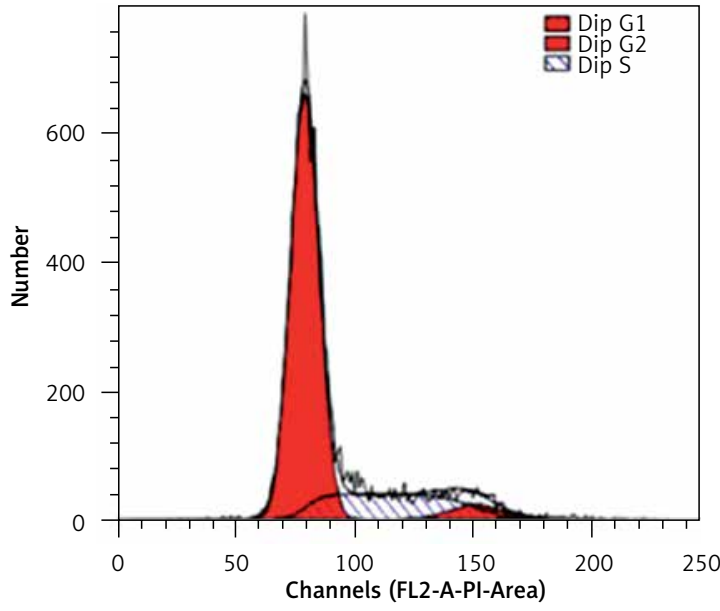

Res + TNF- $\alpha$

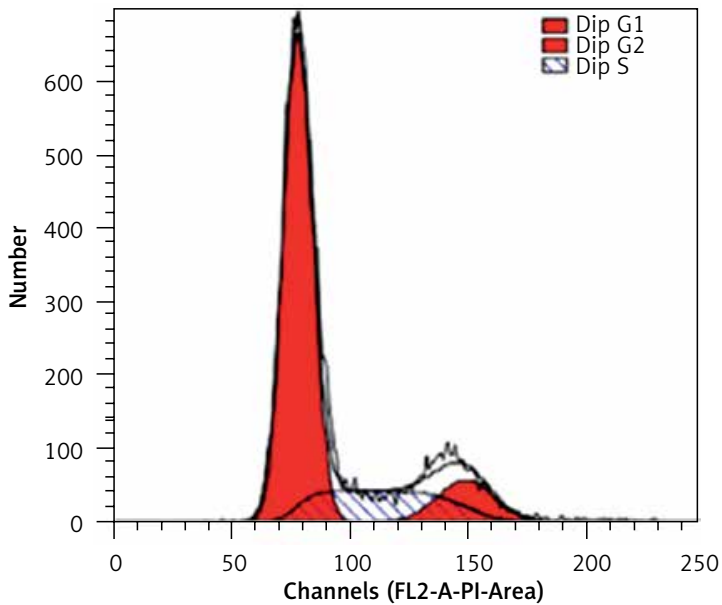

B 0.7

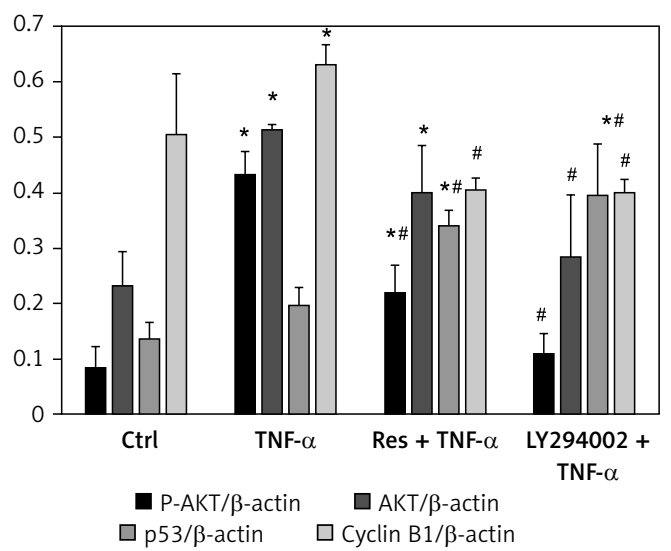

TNF- $\alpha$

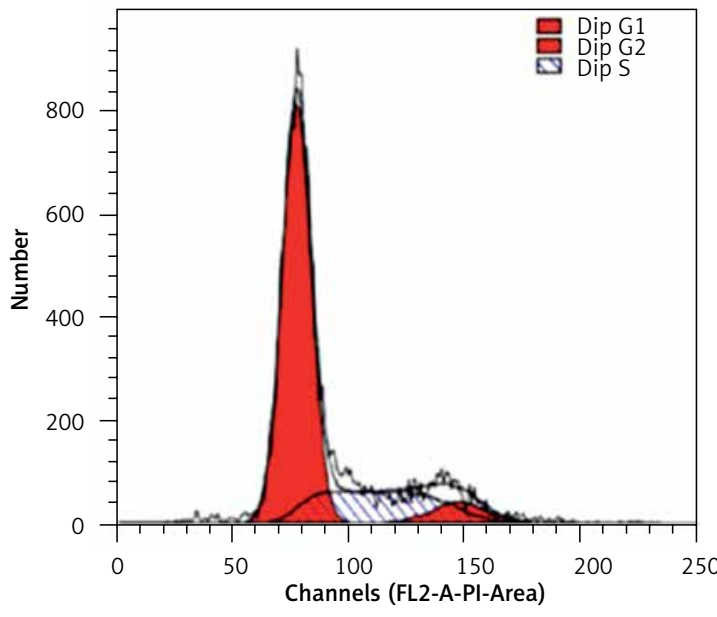

LY294002 + TNF- $\alpha$

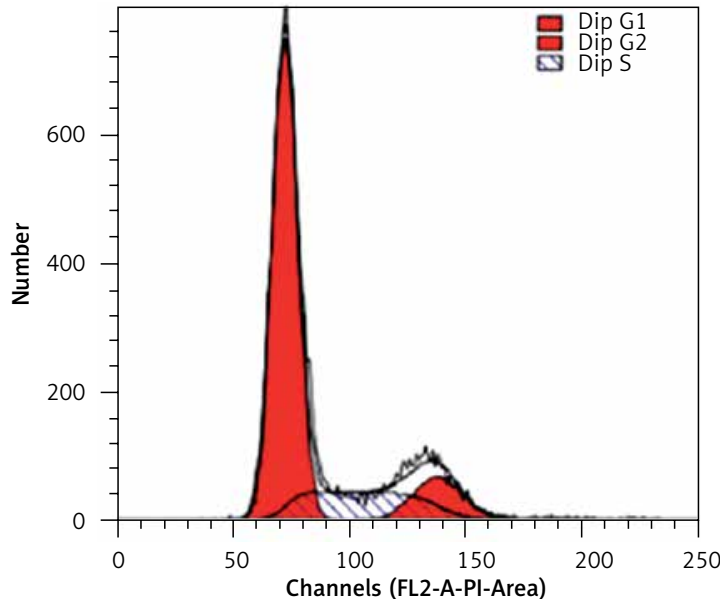

Figure 3. Effects of resveratrol or phosphoinositide 3-kinase (PI3K) inhibitor (LY294002) on the phosphoinositide 3-kinase/serine-threonine kinase (PI3K/AKT) pathway and cell cycle in fibroblast-like synoviocytes in rheumatoid arthritis cells treatment with tumour necrosis factor $\alpha$ (TNF- $\alpha$ ). A - The expression of p-AKT, AKT, p53, and cell cycle-related cycle protein were detected by western blot in different groups. B - Protein expression levels of the indicated proteins were quantitatively analysed by densitometry and normaliesd with $\beta$-actin. $\mathbf{C}-$ Cell cycle was detected by flow cytometry method

${ }^{\star} p<0.05$ compared with control group. ${ }^{*} p<0.05$ compared with TNF- $\alpha$ group. 

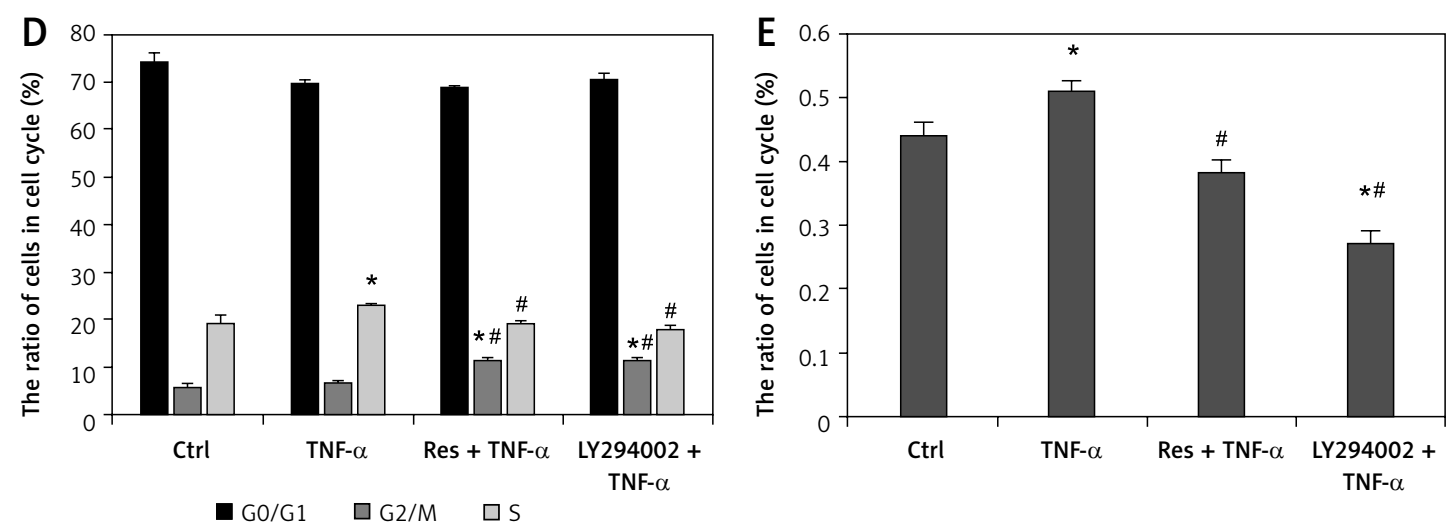

Figure 3. Cont. D - Quantitative analysis of the ratio of cells in different cell cycle stages. E - Cell viability was detected by Cell Counting Kit- 8 assay. Data are expressed as mean \pm SEM of 3 independent experiments ${ }^{*} p<0.05$ compared with control group. ${ }^{*} p<0.05$ compared with TNF- $\alpha$ group.

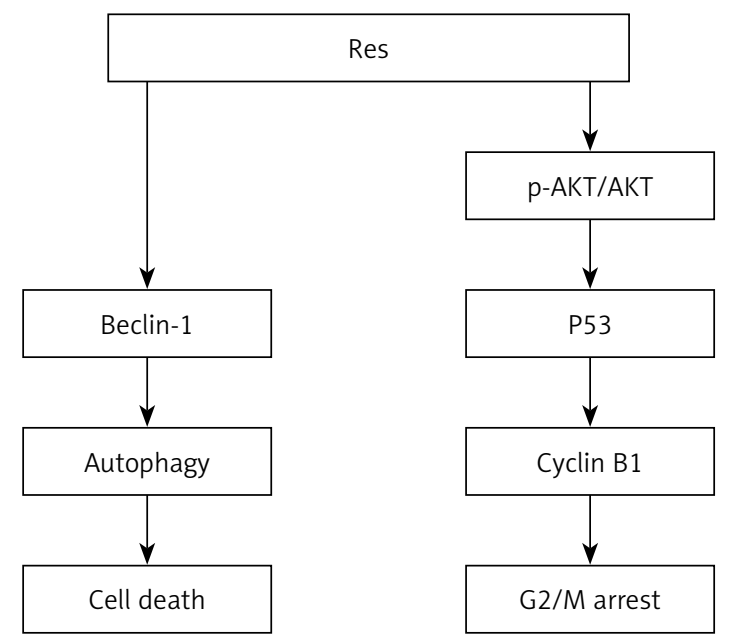

Figure 4. Simplified schematic shows the role and potential molecular mechanism of resveratrol in inhibiting TNF- $\alpha$ induced tumor-like proliferation of RA-FLS. Treatment with resveratrol suppressed cell survival and promoted cell apoptosis by downregulation of TNF- $\alpha$-induced autophagy and upregulation of p53 in RA-FLS

upregulation of p53 [33, 34]. In the present study, we hypothesised that resveratrol, a natural polyphenol compound, could induce cell apoptosis in a p53-dependent manner in RA-FLS. Consistent with this hypothesis, our data showed that resveratrol obviously upregulated tumour suppressor protein $\mathrm{p} 53$ and downregulated cell cycle-related protein cyclin B1 compared with the TNF- $\alpha$-alone group, which further led to cell cycle stagnation at the G2/M phase, and the ratio of cells in S phase was obviously reduced. Our data indicated that resveratrol may regulate $\mathrm{p} 53$ and cell cycle in RA-FLS.

Additionally, we found that TNF- $\alpha$ exposure increased AKT and p-AKT levels in RA-FLS cells compared to controls. However, upon treatment with resveratrol or PI3K inhibitor LY294002, the expression of p-AKT and AKT was obviously decreased, which further led to upregulation of p53 and down- regulation of cyclin B1, resulting in cells arrested at G2/M stage, and the ratio of cells in the $S$ phase was decreased. Taken together, these findings suggested that resveratrol suppressed RA-FLS cell survival and promoted cell apoptosis partly through the AKT-p53-cyclin B1 axis. Due to the small number of patients $(n=6)$ in the present study, the results require confirmation in further research.

In conclusion, our preliminary study demonstrates for the first time that resveratrol plays an important role in abolishing the effect of TNF- $\alpha$-mediated apoptosis resistance in RA synovial fibroblasts. Resveratrol reversed TNF- $\alpha$-induced cell survival and triggered cell apoptosis in RA-FLS at least partly through downregulation of Beclin-1 expression and inhibition ohe $\mathrm{ftp} 53$-cycin B1 axis, which sheds light on new therapies for RA.

\section{Acknowledgements}

This study was supported by grants from the Hunan Provincial Natural Science Foundation of China (No. 2019JJ50895) and National Natural Science Foundation of China Youth Fund (No. 81302567, No. 81601436).

\section{Conflicts of interests}

The authors declare no conflicts of interest.

\section{References}

1. Calabresi E, Petrelli F, Bonifacio AF, Puxeddu I, Alunno A. One year in review 2018: pathogenesis of rheumatoid arthritis. Clin Exp Rheumatol 2018; 36: 175-84.

2. Yu S, Lu Y, Zong M, Tan Q, Fan L Hypoxia-induced miR191-C/EBP $\beta$ signaling regulates cell proliferation and apoptosis of fibroblast-like synoviocytes from patients with rheumatoid arthritis. Arthritis Res Ther 2019; 21: 78.

3. Gu X, Gu B, Lv X, et al. 1, 25-dihydroxy-vitamin D3 with tumor necrosis factor-alpha protects against rheumatoid arthritis by promoting p53 acetylation-mediated apoptosis via Sirt1 in synoviocytes. Cell Death Dis 2016; 7: e2423. 
4. Conigliaro P, Triggianese P, De Martino E, et al. Challeng es in the treatment of rheumatoid arthritis. Autoimmun Rev 2019; 18: 706-13.

5. Tłustochowicz M, Śliwczyński AM, Brzozowska, M, Teter $Z$, Marczak M. Sequentiality of treatment in the rheumatoid arthritis drug programme in the years 20092014. Arch Med Sci 2018; 14: 569-71.

6. Jiang Z, Chen K, Cheng L, et al. Resveratrol and cancer treatment: updates. Ann NY Acad Sci 2017; 1403: 59-69.

7. Monteillier A, Voisin A, Furrer P, Allémann E, Cuendet M Intranasal administration of resveratrol successfully prevents lung cancer in A/J mice. Sci Rep 2018; 8 : 14257.

8. Liu Y, Tong L, Luo Y, Li X, Chen G, Wang Y. Resveratrol inhibits the proliferation and induces the apoptosis in ovarian cancer cells via inhibiting glycolysis and targeting AMPK/mTOR signaling pathway. J Cell Biochem 2018; 119: 6162-72.

9. Hafner A, Bulyk ML, Jambhekar A, Lahav G. The multiple mechanisms that regulate $\mathrm{p} 53$ activity and cell fate. Nat Rev Mol Cell Biol 2019; 20: 199-210.

10. Aubrey BJ, Kelly GL, Janic A, Herold MJ, Strasser A. How does p53 induce apoptosis and how does this relate to p53-mediated tumour suppression? Cell Death Differ 2018; 25: 104-13.

11. Vanamala J, Reddivari L, Radhakrishnan S, Tarver C. Resveratrol suppresses IGF-1 induced human colon cancer cell proliferation and elevates apoptosis via suppression of IGF-1R/Wnt and activation of p53 signaling pathways. BMC Cancer 2010; 10: 238.

12. Parida PK, Mahata B, Santra A, et al. Inhibition of cancer progression by a novel trans-stilbene derivative through disruption of microtubule dynamics, driving G2/M arrest, and p53-dependent apoptosis. Cell Death Dis 2018; 9: 448.

13. Hernandez-Valencia J, Garcia-Villa E, Arenas-Hernandez A, Garcia-Mena J, Diaz-Chavez J, Gariglio P. Induction of p53 phosphorylation at serine 20 by resveratrol is required to activate p53 target genes, restoring apoptosis in MCF-7 cells resistant to cisplatin. Nutrients 2018; 3; 10: 1148.

14. Yu CL, Yang SF, Hung TW, Lin CL, Hsieh YH, Chiou HL. In hibition of elF2 $\alpha$ dephosphorylation accelerates pterostilbene-induced cell death in human hepatocellular carcinoma cells in an ER stress and autophagy-dependent manner. Cell Death Dis 2019; 10: 418.

15. Zhang B, Yin X, Sui S. Resveratrol inhibited the progression of human hepatocellular carcinoma by inducing autophagy via regulating p53 and the phosphoinositide 3-kinase/protein kinase B pathway. Oncol Rep 2018; 40: 2758-65.

16. Arnett FC, Edworthy SM, Bloch DA, et al. The American Rheumatism Association 1987 revised criteria for the classification of rheumatoid arthritis. Arthritis Rheum 1988; 31: 315-24.

17. Li S, Chen JW, Xie X, et al. Autophagy inhibitor regulates apoptosis and proliferation of synovial fibroblasts through the inhibition of PI3K/AKT pathway in collagen-induced arthritis rat model. Am J Transl Res 2017; 9: 2065-76

18. Shintani T, Klionsky DJ. Autophagy in health and disease: a double-edged sword. Science 2004; 306: 990-5.

19. Zhang T, Li H, Shi J, et al. p53 predominantly regulates IL-6 production and suppresses synovial inflammation in fibroblast-like synoviocytes and adjuvant-induced arthritis. Arthritis Res Ther 2016; 18: 271.
20. Xiao P, Hao Y, Zhu X, Wu X. p53 contributes to quercetin-induced apoptosis in human rheumatoid arthritis fibroblast-like synoviocytes. Inflammation 2013; 36: 272-8.

21. Leyva-Porras C, Saavedra-Leos MZ, Cervantes-González E, Aguirre-Bañuelos P, Silva-Cázarez MB, Álvarez-Salas C. Spray drying of blueberry juice-maltodextrin mixtures: evaluation of processing conditions on content of resveratrol. Antioxidants (Basel) 2019; 8: 437.

22. García-Quiroz J, García-Becerra R, Santos-Cuevas C, et al. synergistic antitumorigenic activity of calcitriol with curcumin or resveratrol is mediated by angiogenesis inhibition in triple negative breast cancer xenografts. Cancers (Basel) 2019; 11: 1739.

23. Jasso-Miranda C, Herrera-Camacho I, Flores-Mendoza LK, et al. Antiviral and immunomodulatory effects of polyphenols on macrophages infected with dengue virus serotypes 2 and 3 enhanced or not with antibodies. Infect Drug Resist 2019; 12: 1833-52.

24. Bektas A, Schurman SH, Gonzalez-Freire $M$, et al. Age-associated changes in human CD4+ $T$ cells point to mitochondrial dysfunction consequent to impaired autophagy. Aging (Albany NY) 2019; 11: 9234-63.

25. Pandurangan AK, Ismail S, Esa NM, Munusamy MA, et al. Inositol-6 phosphate inhibits the mtor pathway and induces autophagy-mediated death in ht-29 colon cancer cells. Arch Med Sci 2018; 14: 1281-88.

26. Mizushima N, Komatsu M. Autophagy: renovation of cells and tissues. Cell 2011; 147: 728-41.

27. Yang $H, N i$ HM, Ding WX. The double-edged sword of MTOR in autophagy deficiency induced-liver injury and tumorigenesis. Autophagy 2019; 15: 1671-3.

28. Mirza-Aghazadeh-Attari M, Mohammadzadeh A, Adib $A$, et al. Melatonin-mediated regulation of autophagy: making sense of double-edged sword in cancer. J Cell Physiol 2019; 234: 17011-22.

29. Das CK, Mandal M, Kögel D. Pro-survival autophagy and cancer cell resistance to therapy. Cancer Metastasis Rev 2018; 37: 749-66.

30. Dinesh P, Rasool M. Berberine mitigates IL-21/IL-21R mediated autophagic influx in fibroblast-like synoviocytes and regulates Th17/Treg imbalance in rheumatoid arthritis. Apoptosis 2019; 24: 644-61.

31. Chen YM, Chang CY, Chen $\mathrm{HH}$, et al. Association between autophagy and inflammation in patients with rheumatoid arthritis receiving biologic therapy. Arthritis Res Ther 2018; 20: 268.

32. Igarashi $H$, Hirano $H$, Yahagi A, Saika T, Ishihara K. Anti-apoptotic roles for the mutant $\mathrm{p} 53 \mathrm{R} 248 \mathrm{Q}$ through suppression of p53-regulated apoptosis-inducing protein 1 in the RA-derived fibroblast-like synoviocyte cell line MH7A. Clin Immunol 2014; 150: 12-21.

33. Zhao H, Han L, Jian Y, et al. Resveratrol induces apoptosis in human melanoma cell through negatively regulating Erk/PKM2/Bcl-2 axis. Onco Targets Ther 2018; 11: 8995-9006.

34. Cheah FK, Leong KH, Thomas NF, Chin HK, Ariffin A, Awang K. Resveratrol analogue, (E)-N-(2-(4-methoxystyryl) phenyl) furan-2-carboxamide induces $\mathrm{G} 2 / \mathrm{M}$ cell cycle arrest through the activation of p53-p21CIP1/WAF1 in human colorectal HCT116 cells. Apoptosis 2018; 23: 329-42. 